\title{
Erratum to Combined microvascular breast and lymphatic reconstruction with deep inferior epigastric perforator flap and gastroepiploic vascularized lymph node transfer for postmastectomy lymphedema patients
}

doi: $10.21037 /$ gs-2020-01e

View this article at: http://dx.doi.org/10.21037/gs-2020-01e

Erratum to: Gland Surg 2020;9(2):512-520

Combined microvascular breast and lymphatic reconstruction with deep inferior epigastric perforator flap and gastroepiploic vascularized lymph node transfer for postmastectomy lymphedema patients

In April 2020 issue of Gland Surgery, the article entitled "Combined microvascular breast and lymphatic reconstruction with deep inferior epigastric perforator flap and gastroepiploic vascularized lymph node transfer for postmastectomy lymphedema patients" (1) was published with some errors.

Due to editor's mistake during the proof process, the Figure 3 and Figure 4 did not present completely, we here present the two complete pictures below.
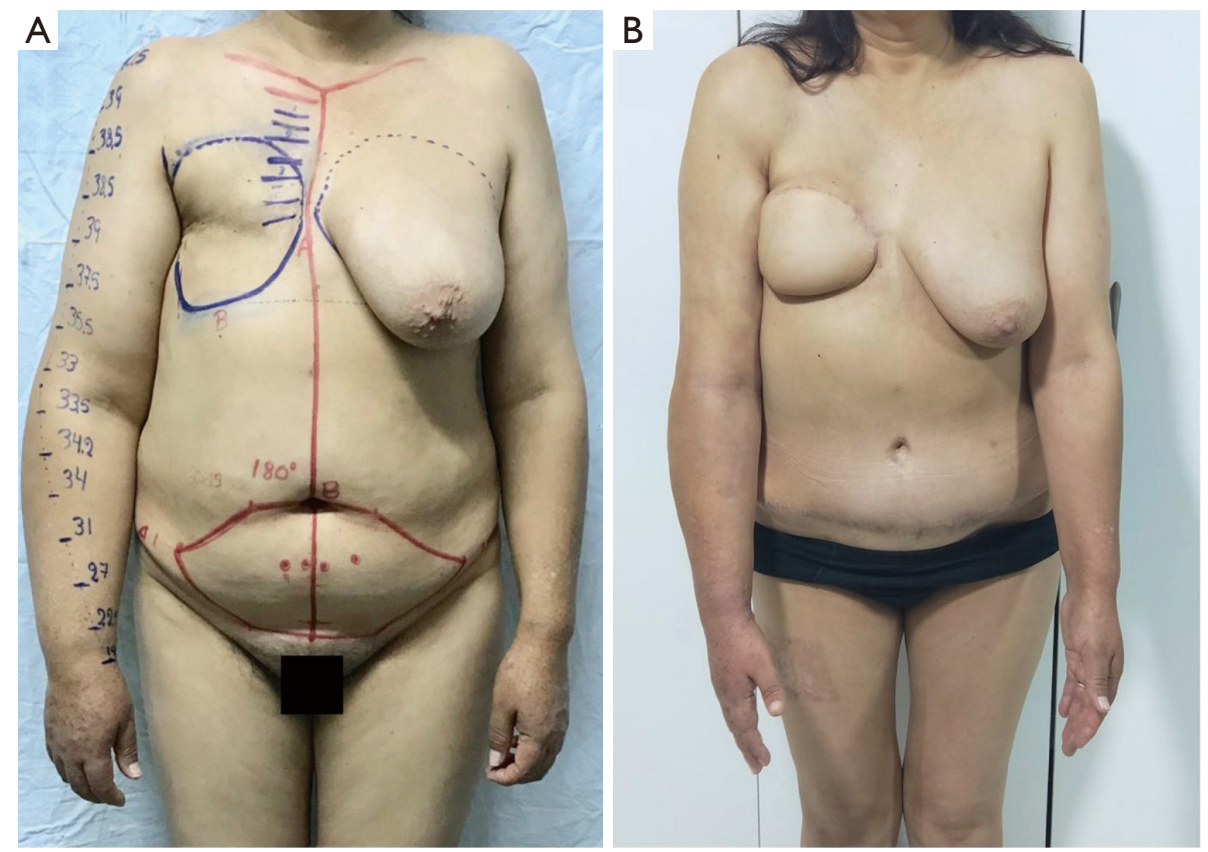

Figure 3 A 49-year-old female patient with left breast cancer-related lymphedema (BCRL) with 12 months of complex decongestive therapy (CDT) without significant improvement. The patient underwent the combined microvascular autologous breast reconstruction and gastroepiploic vascularized lymph node flap transfer. (A) Preoperative picture. Notice the mastectomy scar over her left chest and the volume and size discrepancy between the upper extremities. (B) Postoperative picture at 12 -months follow-up evidencing a mean limb circumference reduction rate of $30 \%$. In addition, the patient reported improved lymphedema-associated symptoms. 

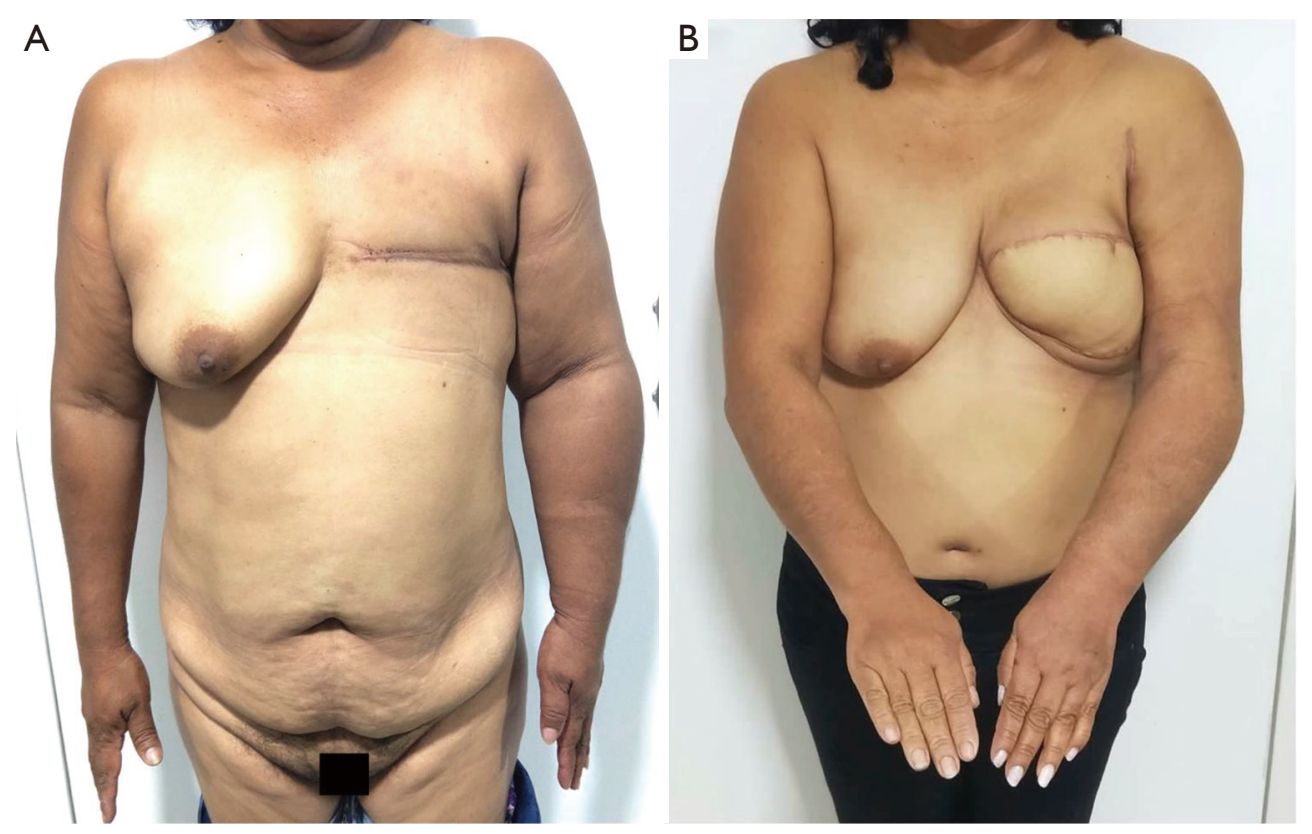

Figure 4 Pre and postoperative pictures of a 45-year-old female patient with right breast cancer-related lymphedema (BCRL). She had received 15 months of complex decongestive therapy with unsatisfactory results. (A) Preoperative picture. (B) Postoperative picture at 12 months of follow-up showing a limb circumference reduction rate of $39 \%$.

The publisher regrets the error and is sorry for the inconvenience caused.

\section{References}

1. Ciudad P, Manrique OJ, Bustos SS, et al. Combined microvascular breast and lymphatic reconstruction with deep inferior epigastric perforator flap and gastroepiploic vascularized lymph node transfer for postmastectomy lymphedema patients.

Gland Surg 2020;9:512-20.

Cite this article as: Erratum to Combined microvascular breast and lymphatic reconstruction with deep inferior epigastric perforator flap and gastroepiploic vascularized lymph node transfer for postmastectomy lymphedema patients. Gland Surg 2020;9(3):867-868. doi: 10.21037/gs-2020-01e 